$\dagger$ Presently at Bowling Green State University, Bowling Green, Ohio.

${ }^{1}$ P. E. Wigen, C. F. Kooi, M. R. Shanabarger, and T. D. Rossing, Phys. Rev. Letters 9, 206 (1962).

${ }^{2}$ A. M. Portis, Appl. Phys. Letters 2, 69 (1963).

${ }^{3}$ C. F. Kooi, P. E. Wigen, M. R. Shanabarger, and J. V. Kerrigan, J. Appl. Phys. 35, 791 (1964).

${ }^{4}$ M. Sparks, Phys. Rev. Letters 22, 1111 (1969).

${ }^{5}$ M. Sparks, Ferromagnetic-Relaxation Theory
(McGraw-Hill, New York, 1964).

${ }^{6} \mathrm{M}$. Sparks, to be published.

${ }^{7}$ P. Pincus, Phys. Rev. 118, 658 (1960).

${ }^{8} \mathrm{M}$. Sparks, to be published.

${ }^{9} \mathrm{P}$. Wigen, to be published.

${ }^{10} \mathrm{E}$. Schlömann, to be published.

${ }^{11}$ G. I. Lykken, Phys. Rev. Letters 19, 1431 (1967).

${ }^{12}$ C. E. Patton and C. H. Wilts, J. Appl. Phys. $\underline{38}$, 3537 (1967).

\title{
NUCLEAR GIANT QUADRUPOLE RESONANCES IN SPHERICAL EVEN-EVEN NUCLEI*
}

\section{Tadas D. Urbas and Walter Greiner}

Institut für Theoretische Physik der Universität Frankfurt am Main, 6 Frankfurt am Main 1, Germany (Received 5 March 1970)

\begin{abstract}
The dynamic collective model has been extended to quadrupole giant resonances in spherical nuclei. The splitting of giant dipole and giant quadrupole resonances due to their coupling to surface vibrations has been calculated for Sn isotopes. Agreement with recent $\gamma$-absorption measurements of the Livermore group has been found.
\end{abstract}

The dynamic collective theory was successful in explaining the main structure of giant dipole resonances in deformed and spherical nuclei. ${ }^{1,2}$ In this Letter it has been extended to include the coupling of the quadrupole giant resonance and the surface vibrations of medium heavy and heavy spherical even-even nuclei. The total Hamiltonian can be written

$$
H=H_{s}+\sum_{\lambda=1,2} H_{\lambda}
$$

where $H_{s}$ is the harmonic Hamiltonian of the surface quadrupole vibrations. $H_{\lambda}(\lambda=1,2)$ denote as well the Hamiltonian for the giant dipole $(\lambda=1)$ and giant quadrupole $(\lambda=2)$ oscillations as well as their interaction with the low-energy surface vibrations:

$$
\begin{aligned}
H_{\lambda}=( & -)^{\lambda}(2 \lambda+1)^{1 / 2} \hbar \Omega_{\lambda}\left\{\left[q^{[\lambda] \dagger} \times q^{[\lambda]}\right]^{[0]}+\frac{1}{2}(-)^{\lambda}(2 \lambda+1)^{1 / 2}\right\} \\
& +\hbar \Omega_{\lambda} A_{\lambda}\left\{\left[q^{\left[\lambda{ }^{\dagger}\right.} \times q^{[\lambda]^{\dagger}} \times \alpha^{[2]}\right]^{[0]}+\left[q^{[\lambda]} \times q^{[\lambda]} \times \alpha^{[2]}\right]^{[0]}\right\}+\hbar \Omega_{\lambda} B_{\lambda}\left[q^{\left[\lambda{ }^{\dagger}\right.} \times q^{[\lambda]} \times \alpha^{[2]}\right]^{[0]} \\
& +\hbar \Omega_{\lambda} \sum_{\sigma=0,2,4} A_{\lambda \sigma}\left\{\left[q^{[\lambda]^{\dagger}} \times q^{[\lambda]^{\dagger}} \times\left[\alpha^{[2]} \times \alpha^{[2]}\right]^{[\sigma]}\right]^{[0]}+\left[q^{[\lambda]^{\dagger}} \times q^{[\lambda]} \times\left[\alpha^{[2]} \times \alpha^{[2]}\right]^{[\sigma]}\right]^{[0]}\right\} \\
& \left.+\hbar \Omega_{\lambda} \sum_{\sigma=0,2,4} B_{\lambda \sigma}\left\{\left[q^{[\lambda]} \times q^{[\lambda]} \times\left[\alpha^{[2]} \times \alpha^{[2]}\right]^{[\sigma]}\right]^{[0]}\right\}+\delta_{\sigma 0}(-)^{\lambda}(2 \lambda+1)^{1 / 2}\left[\alpha^{[2]} \times \alpha^{[2]}\right]^{[0]}\right\} .
\end{aligned}
$$

Here $q^{[\lambda]^{\dagger}}$ and $q^{[\lambda]}$ are spherical creation and annihilation operators for the giant multipole resonance phonons. $\alpha^{[2]}$ denote the amplitudes for the surface quadrupole vibrations which are expressed in terms of creation and annihilation phonon operators by $\alpha^{[2]}=[\hbar /(2 B \omega)]^{1 / 2}\left(\beta^{[2] \dagger}+\beta^{[2]}\right) . \hbar \omega$ $=\hbar(C / B)^{1 / 2}$ is the energy of the surface vibrations and $B$ the corresponding mass parameter. Both are deduced from the first excited $2^{+}$state of the nucleus and the transition probability $B\left(E 2,2^{+}\right.$ $-0^{+}$) which is related to the mean-square amplitude of the surface vibrations $\beta_{0}{ }^{2}: B\left(E 2,2^{+} \rightarrow 0^{+}\right)$ $=\left[\left(\frac{3}{4} \pi\right) Z R_{0}{ }^{2}\right]^{2} \beta_{0}{ }^{2}$. $\hbar \Omega_{1}$ is the energy of the unperturbed giant dipole resonance and is given by

$$
\hbar \Omega_{1}=\frac{2.08 \hbar}{R_{0}}\left(\frac{8 \kappa}{M^{*}} \frac{N Z}{A^{2}}\right)^{1 / 2} .
$$

It contains the symmetry-energy parameter $\kappa$ of the mass formula and the effective mass of the nucleon, $M^{*}=M /(1+\alpha)$. According to the hydrodynamical model the giant dipole and giant quadrupole energies are related to each other, $\hbar \Omega_{2}=1.6 \hbar \Omega_{1} \cdot{ }^{3}$ The coupling constants $A_{\lambda}, B_{\lambda}$, $A_{\lambda \sigma}$, and $B_{\lambda \sigma}$ are obtained by solving the boundary-value problem of the hydrodynamical model. ${ }^{4,5}$ They are $A_{1}=1.5088, B_{1}=-1.5795, A_{10}$ $=0.9881, B_{10}=1.4024, A_{12}=0.9503, B_{12}=-1.7753$, $A_{14}=B_{14}=0, A_{2}=-1.4376, B_{2}=1.1914, A_{20}=0.8676$, $B_{20}=0.6686, A_{22}=3.9341, B_{22}=-1.4494, A_{24}$ $=3.9011, B_{24}=-0.0188$.

The Hamiltonian (1) is diagonalized in the function space of the harmonic dipole and quadrupole oscillations. Due to the interaction contained in 

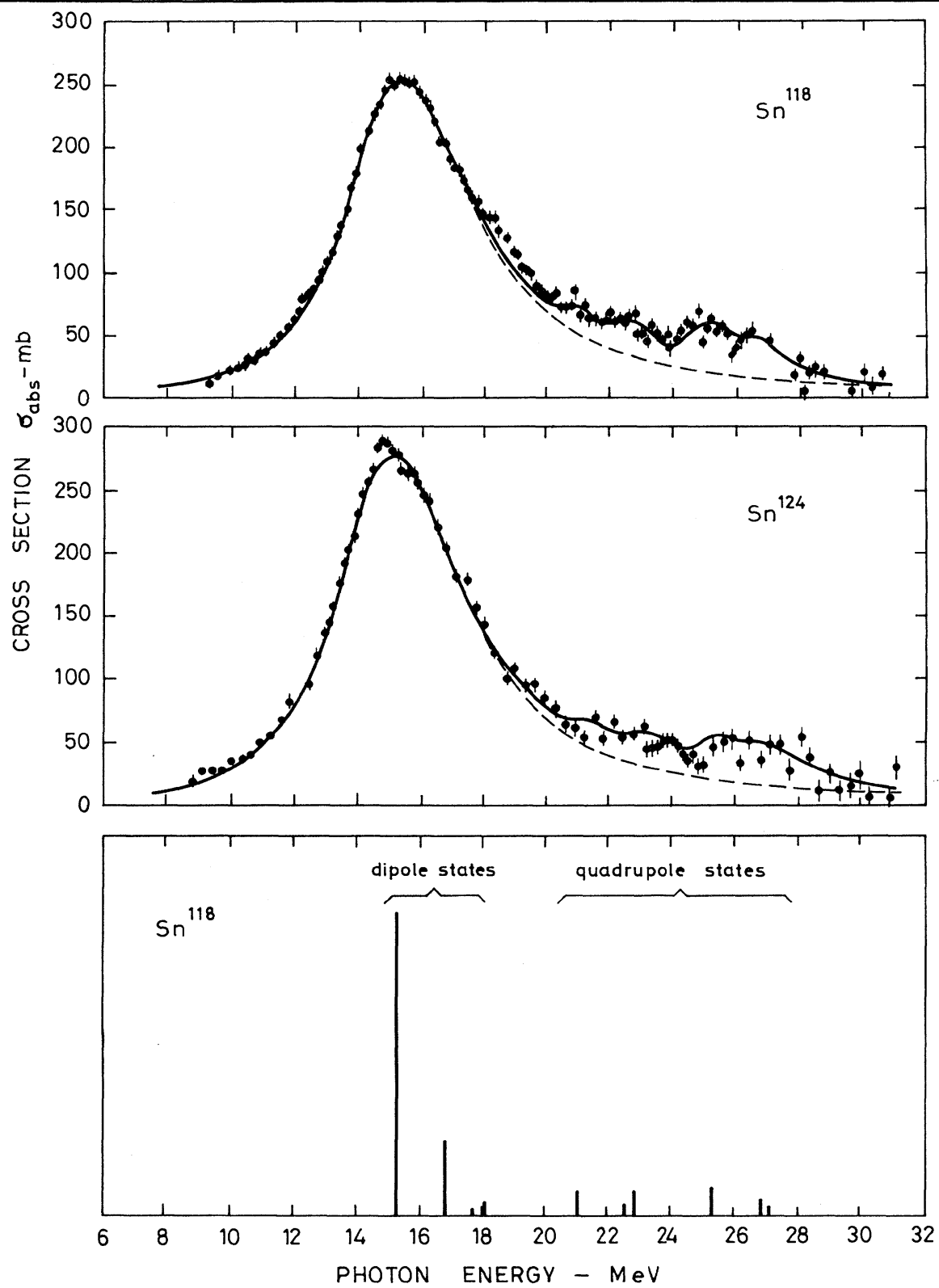

FIG. 1. The photon absorption cross section as predicted by the dynamic collective theory (full line) compared with recent $\gamma$-absorption measurements of the Livermore group (see Ref. 6). The lower part of the figure shows energies and strengths of the dipole and quadrupole giant resonances. The splitting of these resonances due to their coupling to surface vibrations is evident.

(2) a spreading of the dipole and quadrupole strength occurs. This is shown for the isotopes $\mathrm{Sn}^{118}$ and $\mathrm{Sn}^{124}$ in the lower part of Fig. 1. The occuring structure resembles to some extent that of strongly deformed nuclei ${ }^{2}$

The $\gamma$-absorption cross sections shown in Figs. 1 and 2 are obtained by summing the contributions of the different resonances. Lorentzian shapes with a common width for the giant dipole states, $\Gamma_{D}$, and for the giant quadrupole states, $\Gamma_{\mathrm{Q}}$, are assumed (see Table $\mathrm{I}$ ). The experimental data are those recently obtained by the Liver- more group ${ }^{6}$ measuring the $\gamma$-absorption cross sections with monochromatic $\gamma$ rays obtained from electron-positron annihilation in flight. These data seem to reveal the theoretically predicted structure of the quadrupole giant resonances. Both the energies and the strengths of the various levels are indicated in the lower part of Fig. 1. The agreement with the observed structure in the excess cross section at the highenergy tail of the dipole giant resonance is surprising. The slight substructure in the giant dipole resonance at about 16.5 and $18.5 \mathrm{MeV}$ can 


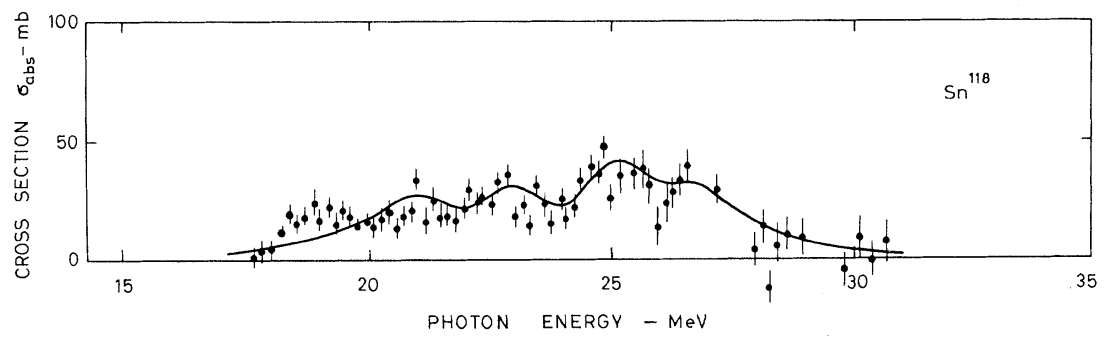

FIG. 2. The experimental excess cross section (see Ref。6) over the theoretical giant dipole tail compared with the theoretical quadrupole absorption cross section (full curve).

possibly be due to the vibrational giant dipole satellites predicted at about these energies (see the lower part of Fig。1)。In fact, it has been shown ${ }^{7}$ that the escape widths of the giant dipole satellites can vary quite appreciably. Nevertheless, more experimental evidence is necessary

Table I. The various parameters for the Sn isotopes. $\hbar \Omega_{1}$ and $\hbar \Omega_{2}$ denote the unperturbed giant dipole and giant quadrupole energies, respectively. $\hbar \omega$ is the energy of the first $2^{*}$ surface phonon, $\beta_{0}$ is the mean deformation, and $\Gamma_{D}$ and $\Gamma_{\mathrm{Q}}$ are the assumed widths of the giant dipole and giant quadrupole resonances, respectively。 $\alpha$ is the parameter for the effective mass.

\begin{tabular}{lcl}
\hline & $\mathrm{Sn}^{118}$ & \\
$\hbar \Omega_{1}=15.6 \mathrm{MeV}$ & & $\Gamma_{D}=4.8 \mathrm{MeV}$ \\
$\hbar \omega=1.230 \mathrm{MeV}$ & $\alpha=0.2$ & $\Gamma_{Q}=2 \mathrm{MeV}$ \\
$\beta_{0}=0.116$ & & \\
$\hbar \Omega_{2}=25.0 \mathrm{MeV}$ & & \\
& $\mathrm{Sn}^{124}$ & \\
$\hbar \Omega_{1}=15.2 \mathrm{MeV}$ & & $\Gamma_{D}=4.8 \mathrm{MeV}$ \\
$\hbar \omega=1.131 \mathrm{MeV}$ & $\alpha=0.2$ & $\Gamma_{Q}=2 \mathrm{MeV}$ \\
$\beta \omega_{0}=0.108$ & & \\
$\hbar \Omega_{2}=24.4 \mathrm{MeV}$ & & \\
\hline
\end{tabular}

to confirm the interpretation of this structure as quadrupole giant resonances. The electron excitation of these resonances in medium heavy nuclei should be especially valuable. ${ }^{8}$

\footnotetext{
${ }^{*}$ Work supported by the Bundesministerium für wissenschaftliche Forschung and by the Deutsche Forschungsgemeinschaft .

${ }^{1}$ M. Danos and W. Greiner, Phys. Rev. 134, B284 (1964); J. Le Tourneux, Kgl. Danske Videnskab. Selskab, Mat.-Fys. Medd. 34, No. 11 (1965); M. Huber, M. J. Weber, M. Danos, and W. Greiner, Phys. Rev. Letters 15, 529 (1965); H. J. Weber, M. Huber, and W. Greiner, Z. Physik 192, 182 (1966), and 223 (1966).

${ }^{2}$ R. Ligensa and W. Greiner, Nucl. Phys. A92, 673 (1967).

${ }^{3}$ J. M. Eisenberg and W. Greiner, "Nuclear Models" (North-Holland, Amsterdam, to be published).

${ }^{4}$ T. Urbas and W. Greiner, Z. Physik 196, 44 (1966).

${ }^{5}$ H. Stock and H. Arenhövel, Z. Physik 212, 83 (1968).

${ }^{6}$ S. C. Fultz, R. L. Bramblett, B. L. Berman, J. T. Caldwell, and M. A. Kelly, Lawrence Radiation Laboratory Report No. UCRL-71512 (to be published), and Phys. Rev. 186, 1255 (1970).

${ }^{7}$ R. Ligensa and W. Greiner, Ann. Phys. (N.Y.), 51, 28 (1969).

${ }^{8}$ D. Drechsel, Z. Physik 192, 81 (1966)。
}

\section{SINGLET DEUTERON EMISSION IN A $\left(\mathrm{He}^{3}, \ddot{d}\right)$ REACTION DESCRIBED BY DISTORTED-WAVE BORN APPROXIMATION}

W. Bohne, M. Hagen, H. Homeyer, ${ }^{*}$ H. Lettau, K. H. Maier, H. Morgenstern, and J. Scheer Hahn-Meitner-Institut für Kernforschung Berlin, Sektor Kernphysik, Berlin-West, Germany (Received 29 December 1969)

Emission of singlet deuterons was observed in the reaction $\mathrm{B}^{10}\left(\mathrm{He}^{3}, \not\right) \mathrm{C}^{11}$ by studying neutron-proton correlations. The resulting angular distributions are very similar to those of triplet deuterons and can be described in distorted-wave Born approximation assuming the $d$ to behave like a single particle.

The mechanism of a nuclear reaction yielding three particles may be dominated by final-state interaction between two of them, which in the case of the neutron-proton system leads to an enhancement of the cross section at small relative energies, related to the existence of a bound state $(-2.23 \mathrm{MeV}$, triplet, $S=1, T=0)$ and an antibound state $(-0.06$, singlet, $S=0, T=1$, sometimes erroneously referred to as a resonant state). The singlet interaction being dominant at 